Check for updates

Cite this: RSC Adv., 2018, 8, 34603

Received 26th July 2018

Accepted 1st October 2018

DOI: $10.1039 / c 8 r a 06324 a$

rsc.li/rsc-advances

\section{Amphiphilic polypeptides with prolonged enzymatic stability for the preparation of self- assembled nanobiomaterials}

\author{
Irina Tarasenko, ${ }^{a}$ Natalia Zashikhina, ${ }^{a b}$ Ivan Guryanov, (D) *b Maria Volokitina, ${ }^{a}$ \\ Barbara Biondi, ${ }^{c}$ Stefano Fiorucci, ${ }^{d}$ Fernando Formaggio, ${ }^{c}$ Tatiana Tennikova (iD *b \\ and Evgenia Korzhikova-Vlakh iD ab
}

\begin{abstract}
Due to their excellent biocompatibility and biodegradability, polypeptides have emerged as versatile bioinspired scaffolds for the preparation of artificial biomaterials. In order to create self-assembled polypeptide nanoparticles with enhanced stability towards enzymatic degradation, we synthesized a series of random and block polypeptides based on lysine and $\alpha$-aminoisobutyric acid (Aib) by the ringopening polymerization of $\mathrm{N}$-carboxyanhydrides (ROP NCA) of the corresponding amino acids. A conformational analysis carried out by means of FT-IR absorption and CD spectroscopies revealed a noticeable difference between random and block copolymers. In turn, the spatial organization of the polypeptide chains induced the formation of nanostructures of different types. The block copolymers self-assembled in vesicle-like structures, whereas polypeptides with randomly distributed monomers formed micelles. In contrast with the polymers with only natural amino acids, all nanoparticles based on Aib/Lys polypeptides showed strong resistance to proteolytic cleavage. The cytotoxicity and the kinetics of the cellular uptake of the prepared nanostructures were also studied. The results obtained could not only contribute to the understanding of long Aib polypeptide folding and self-assembling, but also pave the way to the design of nanomaterials with finely tuned properties in the fields of drug delivery and tissue engineering.
\end{abstract}

\section{Introduction}

The main goal of drug delivery systems is the safe delivery of a bioactive compound at controlled release rate under physiological conditions by various ways of administration (oral, intravenous, transdermal, etc.). ${ }^{1}$ In recent years, a number of organic, inorganic and hybrid micro- and nanomaterials has been developed..$^{2-5}$ Among these, polymer nanostructures offer the widest variability in properties and morphology.$^{6-8}$ They can differ in chemical and biological functionalities, biodegradation rate, optical characteristics and stimuli-response properties. However, not all the polymers, which are successfully shaped as nanoparticles, meet the requirements, such as biocompatibility and/or biodegradability, needed to consider them as biomaterials. In this regard, self-assembled

${ }^{a}$ Institute of Macromolecular Compounds, Russian Academy of Sciences, 31 Bolshoy pr., 199004 St Petersburg, Russia

${ }^{b}$ Institute of Chemistry, St Petersburg State University, 26 Universitetskij Pr., 198504 Petrodvorets, St Petersburg, Russia. E-mail: ivan.guryanov1@gmail.com; tennikova@mail.ru

${ }^{c}$ Department of Clinical and Experimental Medicine, University of Perugia, 1 Piazza L. Severi, 06132 Perugia, Italy

${ }^{d} I C B$, Padova Unit, CNR, Department of Chemistry, University of Padova, 1 Via Marzolo, 35131 Padova, Italy polypeptide nanostructures were recently shown to be a very promising, bio-inspired class of polymers. ${ }^{9-13}$ The structural diversity of the amino acids and the possibility of their different combinations are suitable for designing and producing polypeptides endowed with the desired physical and chemical properties, such as hydrophilic/hydrophobic balance, folding motifs, recognition ability, biocompatibility and biodegradability. ${ }^{14}$ Moreover, the insertion of $\mathrm{D}$ - $\alpha$-amino acids and other non-coded or unnatural residues into the polypeptide backbone enables the fabrication of macromolecules, which are stable to the enzymatic degradation.

One of the most extensively studied, non-coded $\alpha$-amino acids is $\alpha$-aminoisobutyric acid. It does occur in Nature and in particular it characterizes the peptaibiotics, a peptide antibiotic class with membrane permeation abilities. ${ }^{\mathbf{1 5 , 1 6}}$ In addition, its strong tendency to induce helical conformations in peptides is particularly attractive. ${ }^{17-19}$ Aib-rich peptides longer than eight residues tend to adopt the classical $\alpha$-helical conformation, ${ }^{17-22}$ whereas shorter peptides preferentially form $3{ }_{10}$-helices. ${ }^{18,19,23-27}$ Since Aib is not involved in the standard ribosomal biosynthesis, Aib-containing peptides display a remarkable stability to biodegradation. ${ }^{21}$

A variety of syntheses and studies of relatively short Aibcontaining peptides is currently available in the literature. 
Few representative examples are the peptides (Aib-Arg-Aib-Leu $)_{n}$ $(n=1-4),{ }^{21}$ (Glu-Aib-Leu-Aib) ${ }_{n}$ (MW approx. 2800), ${ }^{22}$ Boc-GpnAib-Xaa-OMe (Boc = tert-butyloxycarbonyl, Gpn = gabapentin, Xaa $=$ Leu or Phe, OMe $=$ methoxy), ${ }^{28} \mathrm{~N}^{\alpha}$-thioacylated (Aib) ${ }_{6}$ (on the surface of a gold surface), ${ }^{29}$ Ser-Aib-Phe-Ser-Aib-Phe-Aib ${ }^{30}$ and Phe-Aib-Leu-Ala/Glu/Lys-Aib-Leu-Phe. ${ }^{26}$ The results from the molecular dynamics study of a series of Ac-(Aib) ${ }_{n}$-NHMe (where Ac, acetyl; $n=5-7,10 ; \mathrm{NHMe}=$ methylamino) were recently published as well. ${ }^{27} \mathrm{~A}$ range of publications reported the synthesis of Aib-containing peptides via solution ${ }^{22,31-33}$ or solid-phase techniques. ${ }^{21,34-37}$ Recently, Tsuchiya and Numata described also the enzymatic synthesis of hydrophobic polypeptides based on Ala and Aib. ${ }^{38}$ On the contrary to the short Aib peptides, the preparation of long polypeptides with multiple Aib residues is complicated because of the sterical hindrance of the Aib residue.

The self-assembling properties of Aib-containing peptides were the subject of many investigations..$^{28-32,39-41}$ In particular, Kimura and co-workers synthesized peptide nanotubes of different size (50-200 nm in diameter and 100-600 nm in length) based on block polypeptides $(\mathrm{Sar})_{m}-b$-(Leu-Aib $)_{n}(m=$ $22-25 ; n=6-10)^{31,39}$ and $\left[(\text { Sar })_{26}\right]_{3}-b$ - $\left.\left[(\text { His })_{2} \text {-(Leu-Aib }\right)_{6}\right] \cdot{ }^{40}$ More recently, Haldar and coworkers demonstrated that Boc-Phe-AibGabu-OMe and Boc-Tyr-Aib-Gabu-OMe (Gabu, $\gamma$-aminobutyric acid) are able to form polydisperse microspheres with a diameter of $c a .750 \mathrm{~nm},{ }^{32}$ while Boc-Leu-Aib-Ser-OMe self-assembles into supramolecular nanotubes able to encapsulate hydrophobic guests. ${ }^{\mathbf{4 1}}$

Stimulated by these findings, we designed and synthesized amphiphilic, random and block copolypeptides of Lys and Aib as a novel biomaterial for various biological applications in drug delivery, tissue engineering, etc. On the contrary to the above-mentioned works, where relatively short peptides were described, we decided to explore the use of the easy and costeffective ring-opening polymerization of $\mathrm{N}$-carboxyanhydrides (ROP NCA) for the preparation of long-chain polypeptides. ${ }^{42}$ Lys was chosen to provide solubility in aqueous media and stability to the formed colloids. Furthermore, these positively charged units can bind to negatively charged membranes through electrostatic interactions with subsequent penetration into the cells by means of unspecific cellular uptake. ${ }^{43}$ In turn, being a hydrophobic amino acid, Aib might induce selfaggregation and formation of nanoparticles in aqueous media when inserted in peptides rich of hydrophilic residues. Moreover, the presence of non-coded amino acid Aib can ensure the enhanced enzymatic stability of the nanoparticles, respect to those containing only protein amino acids.

\section{Experimental}

\section{Materials}

H-Lys(Z)-OH (Z, benzyloxycarbonyl) ( $\geq 99.0 \%), \quad \mathrm{H}-\mathrm{Aib}-\mathrm{OH}$ $(\geq 99.0 \%)$, triphosgene (98\%), hexylamine (99.0\%), $\alpha$-pinene, trifluoromethanesulfonic acid (TFMSA), trifluoroacetic acid (TFA), Cy3 succinimidyl ester, methylthiazolyldiphenyltetrazolium bromide (MTT) and 4',6-diamidine-2'-phenylindole dihydrochloride (DAPI) were purchased from Sigma-
Aldrich (Germany) and used without further purification. Dimethylformamide (DMF), dimethylsulfoxide (DMSO), 1,4dioxane, $n$-hexane, tetrahydrofuran (THF), ethyl acetate, 2,2,2trifluoroethanol (TFE) and diethyl ether were purchased from Vecton Ltd. (Russia). Prior to use, all solvents were purified by distillation and dried using standard procedures. The culture media MEM and Fetal Bovine Serum (FBS) Medium 200 were purchased from Gibco (Thermo Fisher Scientific, Switzerland). HepG2 was an ATCC (USA) product.

\section{Methods}

For the synthesis of the monomer, the magnetic stirrer IKA MSH-basic with controllable heating (Germany) was used. Polymerization was carried out in a thermostatic shaker Unimax1010 Incubator 100 Heidolph (Germany). For NMR analysis a Bruker $400 \mathrm{MHz}$ Avance III instrument (Germany) was utilized and DMSO- $d_{6}$ was used. Gel-permeation chromatography (GPC) was performed using LC-10 Shimadzu system (Japan) supplied with a RID 10-A detector and a Styragel column, HMW 6E, 15-20 $\mu \mathrm{m}, 7.8$ i.d. $\times 300 \mathrm{~mm}$. HPLC experiments were performed on a Shimadzu LC-20 system (Canby, USA). The data was acquired and processed with the LS Solution software (Shimadzu, Japan). The colloids were characterized by measuring the hydrodynamic diameter of the nanoobjects by means of a Malvern Zetasizer Nano-ZS DLS instrument, at a scattering angle of $173^{\circ}$ and $25^{\circ} \mathrm{C}$. TEM images were recorded using EM 902A TEM from Philips (Netherlands). The 400 mesh copper grids were hydrophilized by treatment with nitrogen plasma during 30 min using Bal-Tec Med 020 High Vacuum Coating System. The fluorescent plate reader FLUOstar Omega BMG Labtech (Germany) was employed both for the MTT-assay and for studying the cellular uptake kinetics. Cellular uptake visualization was carried out using an Axio Observer Z1 Carl Zeiss microscope (Germany) equipped with the Axiovision MRm digital camera and the ApoTome filtering device.

Synthesis of NCAs. The NCA monomers were prepared from $\mathrm{H}-\mathrm{Lys}(\mathrm{Z})-\mathrm{OH}$ and $\mathrm{H}-\mathrm{Aib}-\mathrm{OH}$, respectively, according to a literature procedure. ${ }^{44}$ Briefly, a $200 \mathrm{~mL}$ triple-jacketed flask, equipped with a reflux condenser and a thermometer, was charged with $2 \mathrm{~g}$ (8 mmol) of H-Lys(Z)-OH [or $3.1 \mathrm{~g}$ (30 mmol) of H-Aib$\mathrm{OH}$ ] and $100 \mathrm{~mL}$ of dry 1,4-dioxane or tetrahydrofuran, respectively. The mixture was also added with 4 equiv. of $\alpha$-pinene, i.e. $4.4 \mathrm{~g}$ (32 mmol) and $16.3 \mathrm{~g}(120 \mathrm{mmol})$, respectively. The suspension was stirred under dry argon at $60{ }^{\circ} \mathrm{C}$ for the Lys and $50{ }^{\circ} \mathrm{C}$ for the Aib NCA preparations. A solution of triphosgene $(0.8 \mathrm{~g}, 2.7 \mathrm{mmol}$ for Lys and $3.0 \mathrm{~g}, 10 \mathrm{mmol}$ for Aib) in $20 \mathrm{~mL}$ of the reaction solvent was added dropwise under stirring. Three (Lys) and four (Aib) additional aliquots of triphosgene, half equiv each, were added dropwise (each during $30 \mathrm{~min}$ ) to the reaction mixture after $1.5 \mathrm{~h}$ from the reaction beginning. In the case of Lys(Z) NCA, the reaction was stopped after $3 \mathrm{~h}$ (the reaction mixture became clear), but for Aib NCA the synthesis was stopped after $4 \mathrm{~h}$ and the unreacted amino acid was removed by filtration. The solvent was removed by rotary evaporation and the product was precipitated with $n$-hexane by cooling overnight. Then, recrystallization from THF/hexane was 
performed for three times. The final NCAs were dried over $\mathrm{P}_{2} \mathrm{O}_{5}$ in vacuo. The yields for Lys(Z) and Aib NCAs were $85 \%$ and $30 \%$, respectively. The chemical structure and purity of the two NCAs were proved by ${ }^{1} \mathrm{H}$ NMR at $25{ }^{\circ} \mathrm{C}$ in $\mathrm{CDCl}_{3}$ solution: Lys(Z) NCA: $\delta 7.43-7.32(\mathrm{~m}, 5 \mathrm{H}), 6.97(\mathrm{~s}, 1 \mathrm{H}), 5.12$ (s, 2H), 4.97 (s, 1H), 4.32$4.23(\mathrm{t}, J=5.2,1 \mathrm{H}), 3.29-3.14(\mathrm{~m}, 2 \mathrm{H}), 2.03-1.90(\mathrm{~m}, 1 \mathrm{H}), 1.90-$ $1.75(\mathrm{~m}, 1 \mathrm{H}), 1.73-1.28(\mathrm{~m}, 4 \mathrm{H})$; Aib NCA: $\delta 6.59-6.40(\mathrm{~m}, 1 \mathrm{H})$, $1.60(\mathrm{~s}, 6 \mathrm{H})$.

Block copolymerization. To obtain a block copolymer of Lys and Aib, a Lys homo-polymer was first synthesized. The solution of $1.0 \mathrm{~g}$ ( $4.9 \mathrm{mmol})$ of Lys(Z) NCA and $16.5 \mathrm{mg}(0.17 \mathrm{mmol})$ of the initiator hexylamine in $25 \mathrm{~mL}$ of ethyl acetate was incubated at $35{ }^{\circ} \mathrm{C}$ for $72 \mathrm{~h}$. The polymer obtained was precipitated with diethyl ether and recrystallized twice from chloroform/diethyl ether. The final precipitate was filtered and dried in vacuo at room temperature. The yield of the polymer, $\mathrm{N}^{\alpha}$-unprotected $\mathrm{P}$ [Lys(Z)] hexylamide, was $80 \%$.

This polymer, hereafter called $\mathrm{P}[\operatorname{Lys}(\mathrm{Z})]$, was used as macroinitiator for the polymerization of Aib NCA. To this end, $110 \mathrm{mg}(850 \mu \mathrm{mol})$ of Aib NCA were added to 53, 70 or $105 \mathrm{mg}$ $(8.5,11.3$ or $16.9 \mu \mathrm{mol})$ of $\mathrm{P}[\mathrm{Lys}(\mathrm{Z})]$, previously dissolved in 3.0, 3.5 or $4.0 \mathrm{~mL}$, respectively, of purified and dried DMF. Thus, the ratio [Aib NCA]/P[Lys(Z)] was 100, 75 and 50, respectively. The reaction mixtures were incubated at $35{ }^{\circ} \mathrm{C}$ for $72 \mathrm{~h}$. The block copolymers obtained were precipitated with diethyl ether, recrystallized twice from chloroform/diethyl ether and dried in vacuo. The block copolymer yield was $61-65 \%$.

Random copolymerization. To prepare the random Lys/Aib copolymer [P(Lys-co-Aib)], the ratio of Lys(Z) to Aib NCAs was $8 / 1,6 / 1,4 / 1,2 / 1$ and $1 / 1$, respectively. The monomer/initiator (hexylamine) ratio was 250 in all cases. Other conditions and purification methods were the same as those described above for the two homo-polymerization steps. The random copolymer yield varied from 67 to $79 \%$.

Lysine deprotection. To remove the Z-protection from the Lys $\varepsilon$-amino group, the copolymer $(70 \mathrm{mg}$ ) was dissolved in $3 \mathrm{~mL}$ of TFA under stirring and kept for $30 \mathrm{~min}$ in an ice bath. Subsequently, $0.3 \mathrm{~mL}$ of TFMSA were added to the polymer solution and the reaction left to proceed at $22{ }^{\circ} \mathrm{C}$ for $3 \mathrm{~h}$. Then, $5 \mathrm{~mL}$ of DMF were added and the reaction mixture was dialyzed against $0.01 \mathrm{M} \mathrm{HCl}$ during $24 \mathrm{~h}$ and then against water for further $24 \mathrm{~h}$. The purified polymer was lyophilized in vacuo.

Polymer characterization. The molecular weights (MWs) of all polymers were determined by GPC by eluting with a $0.1 \mathrm{M}$ LiBr solution in DMF at $60{ }^{\circ} \mathrm{C}$. Poly(methyl methacrylate) was used as standard. The monomer ratio in the polypeptides was evaluated by quantitative HPLC amino acid analysis after total hydrolysis of the samples. The hydrolysis of $1 \mathrm{mg}$ of polypeptide was carried out in $2 \mathrm{~mL}$ of a $6 \mathrm{M} \mathrm{HCl}$ solution with $0.0001 \%$ phenol, in a vacuo-sealed ampoule during $4 \mathrm{~d}$. The solvent was evaporated, and water was added and evaporated several times to eliminate $\mathrm{HCl}$ until a neutral $\mathrm{pH}$ was reached. The products of the hydrolysis were analyzed by reverse-phase (RP) HPLC using a LCMS-8030 Shimadzu system with triple quadruple mass-spectrometry detection (LC-MS) (all from Shimadzu, Japan), equipped with $2 \times 150 \mathrm{~mm}$ Luna $\mathrm{C}_{18}$ column, packed with $5 \mu \mathrm{m}$ particles. The isocratic elution mode was applied with $0.1 \%$ acetonitrile/ $\mathrm{HCOOH}(5 / 95 \mathrm{wt} \%)$ as eluent with a flow rate of $0.3 \mathrm{~mL} \mathrm{~min}^{-1}$.

The self-assembly of the copolymers was carried out by phase-inversion method. ${ }^{52}$ To this aim polymer solution in DMF was placed into dialysis bag with MWCO 3500 and the bag was immersed into the glass filled with $50 \% \mathrm{DMF}$ in water $(\mathrm{v} / \mathrm{v})$. After $2 \mathrm{~h}$ of dialysis with slow stirring the medium was changed to water. The dialysis against water was carried out for $24 \mathrm{~h}$. During this time water was replaced twice with fresh one. Then the nanoparticles were transferred from dialysis bag to the vial and lyophilized. Before the use the samples were redispersed in $0.1 \mathrm{M}$ sodium citrate buffer $(\mathrm{pH} 3.0)$ with ultrasound probe for $30 \mathrm{~s}$. For biodegradation study and cell experiments, sodium citrate buffer was replaced with $0.01 \mathrm{M}$ PBS ( $\mathrm{pH} 7.4$ ) via ultracentrifugation with VivaSpin (MWCO 3000). Dynamic light scattering (DLS) and TEM (transmission electron microscopy) were performed to determine size and morphology of the prepared particles.

Conformational study. The conformations of the polypeptides in the solid state and in solution were studied by Fourier transform infrared (FT-IR) absorption and circular dichroism (CD) spectroscopies. The FT-IR absorption spectra were recorded in the solid state using the IRAffinity-1 Shimadzu (Japan) instrument. CD spectra were obtained with a Jasco J1500 spectropolarimeter. All spectra were recorded at room temperature using Hellma quartz cells with Suprasil@ windows (optical path-length of $0.02 \mathrm{~cm}$ ) at the bandwidth of $1 \mathrm{~nm}$ and the scan speed of $100 \mathrm{~nm} \mathrm{~min}^{-1}$. The signal-to-noise ratio was improved by accumulating 10 scans. The samples (about $0.25 \mathrm{mg} \mathrm{mL}^{-1}$ ) were dissolved under sonication for $15 \mathrm{~min}$ and filtered before each measurement.

Biodegradation study. The enzymatic stability of the nanoparticles synthesized was tested in $0.01 \mathrm{M}$ PBS, pH 7.4, containing papain. To this end, $0.5 \mathrm{mg}$ of enzyme were put into a $0.5 \mathrm{~mL}$ suspension containing $0.5 \mathrm{mg}$ of nanoparticles in PBS. The experiment was carried out for $35 \mathrm{~d}$. During this time, DLS measurements were fulfilled to monitor the change in the hydrodynamic diameter of the polypeptide particles. A quantitative HPLC analysis of free amino acids was carried out after 14 and $35 \mathrm{~d}$. The commercially available, ultra-short, monolithic column $\mathrm{CIM} \mathrm{SO}_{3}$ disk $(3 \mathrm{~mm} \times 12 \mathrm{~mm}$ i.d. $)$, was used as stationary phase. UV detection was performed at $210 \mathrm{~nm}$. A $0.02 \mathrm{M}$ Na-phosphate buffer, pH 7.0 (eluent A) and a $0.0125 \mathrm{M}$ Na-borate buffer, pH 10.5 (eluent B) were employed as mobile phase. The separation of free Lys and Aib was carried out at a flow rate of $0.5 \mathrm{~mL} \mathrm{~min}{ }^{-1}$ following the gradient program: 0 3 min - eluent A, 3-7 min - eluent B.

Cytotoxicity test. HepG2 cells $\left(8 \times 10^{3}\right.$ cells per well) were cultured in MEM in a 96-well plate $(200 \mu \mathrm{L}$ per well) under humidified atmosphere, with $5 \% \mathrm{CO}_{2}$ at $37{ }^{\circ} \mathrm{C}$. After culturing for $24 \mathrm{~h}$, the medium was replaced with the MEM culture medium containing test materials. HepG2 cells without nanoparticles were used as a control. The viability of adherent cells was determined after culturing for $48 \mathrm{~h}$ using the MTT assay. Briefly, after removing the medium, $100 \mu \mathrm{L}$ of MTT solution (1.0 $\mathrm{mg} \mathrm{mL}^{-1}$ ) in MEM culture medium were added into each well and allowed to react at $37^{\circ} \mathrm{C}$ for $1 \mathrm{~h}$. After careful removal 
of the medium, the MTT-formazan crystals formed by survived cells were dissolved in DMSO $(100 \mu \mathrm{L}$ per well $)$. Then, the plate was incubated for $30 \mathrm{~min}$ at room temperature. The absorbance was measured at $540 \mathrm{~nm}$ and $690 \mathrm{~nm}$ in a multi-well plate reader. The values measured at $540 \mathrm{~nm}$ were subtracted from the values at $690 \mathrm{~nm}$ for background correction. The cell viability was calculated by the following formula: cell viability $(\%)=$ optical density (OD) of the treated cells/OD of the nontreated cells $(n=4)$. The data were plotted as a percent of control samples.

Modification of the nanoparticles with Cy3. $50 \mu \mathrm{L}$ of Cy3 solution in DMSO with concentration of $10 \mathrm{mg} \mathrm{mL} \mathrm{mL}^{-1}$ were introduced into $5 \mathrm{~mL}$ of polymer solution in DMSO with concentration of $1 \mathrm{mg} \mathrm{mL} \mathrm{m}^{-1}$. Then, $100 \mu \mathrm{L}$ of Na-borate buffer (12.5 mM, pH 8.4) were added and the reaction mixture was stirred for $24 \mathrm{~h}$ at $22^{\circ} \mathrm{C}$ in the dark. The excess of the dye was removed by dialysis (MWCO 1000) against water for $48 \mathrm{~h}$ in the dark.

Cellular uptake study. To study the cellular uptake of nanoparticles, HepG2 cells were used. Initially, the cells in $5 \mathrm{~mL}$ $\left(2 \times 10^{5}\right.$ cells $)$ in MEM culture medium were seeded on glass slides in a 6-well plate and cultured for $24 \mathrm{~h}$. Then, the incubation medium was changed with a fresh one, containing $0.03 \mathrm{mg} \mathrm{mL} \mathrm{m}^{-1}$ of fluorescently labeled particles, and the cells were incubated for $4 \mathrm{~h}$. The cells were washed with warm PBS solution twice, fixed with 4\% paraformaldehyde and cover slips mounted with Slow Fade Gold Antifade reagent with DAPI.

Kinetics of nanoparticles accumulation in cells. For the quantitative determination of the nanoparticle uptake in HepG2 cells the following method was used. Initially, HepG2 cells $\left(3 \times 10^{4}\right.$ cells/well) were cultured in MEM in a 96-well plate (200 $\mu \mathrm{L}$ per well) for $24 \mathrm{~h}$. Then, the incubation medium was changed with a fresh one containing $0.06 \mathrm{mg} \mathrm{mL}^{-1}$ of fluorescently labeled nanoparticles. HepG2 cells were incubated at different time intervals with nanoparticle suspensions. Then, the medium was removed, the cells were washed with warm PBS three times and $200 \mu \mathrm{L}$ of lysis buffer solution (TRIS buffer, $\mathrm{pH}$ 7.5, 1\% sodium dodecylsulphate). Fluorescence intensity in cells at different time points was recorded using a fluorescence plate reader. Cells without any treatment were used as a negative control $(n=4)$.

\section{Results and discussion}

\section{Synthesis of the polypeptides}

Ring-opening polymerization of NCA is the most common method for the preparation of long-chain, random or block copolypeptides. Below, we illustrate the strategy for the synthesis of the random and block copolymers based on the Lys and Aib $\alpha$-amino acid units (Scheme 1).

A series of random polypeptides with various amounts of Lys and Aib units was obtained and characterized in terms of molecular weight and composition. The initial ratios of monomers and the characteristics of the polymers are given in Table 1.

The molecular weights of the random Lys(Z)/Aib copolymers were examined using gel-permeation chromatography. It was found that an increase of Aib molar concentration in the polymerization mixture led to a decrease of the polypeptide molecular weight. This result is in agreement with the molecular weights of the monomers, e.g. the molecular weight of Aib NCA is approx. 2.4 times lower than that of Lys(Z) NCA. According to the GPC analysis, also $D$ (dispersity) values diminished with the increase of the Aib amount in the polymerization mixture. To determine the [Lys]/[Aib] ratio in the polypeptides, we carried out their acid hydrolyses and analyzed the amino acid content by quantitative RP-HPLC. It was found that an increase of the Aib molar concentration in the polymerization mixture favors the increase of the Aib amount in the final polypeptide (Table 1). A good correlation between calculated and experimentally determined monomer compositions was observed.

In the case of the block copolymers, the polymerization included the preparation of the $[\operatorname{Lys}(\mathrm{Z})]_{n}$ polypeptide and its use as the macroinitiator for the synthesis of $\mathrm{PAib}_{m}-b$-[PLys(Z) $]_{n}$ block sequences. The GPC analysis of $[\operatorname{Lys}(\mathrm{Z})]_{n}$ showed the formation of a quite narrow-dispersed polymer with weight average molecular weight $\left(M_{\mathrm{w}}\right)=7700$ and number average

A
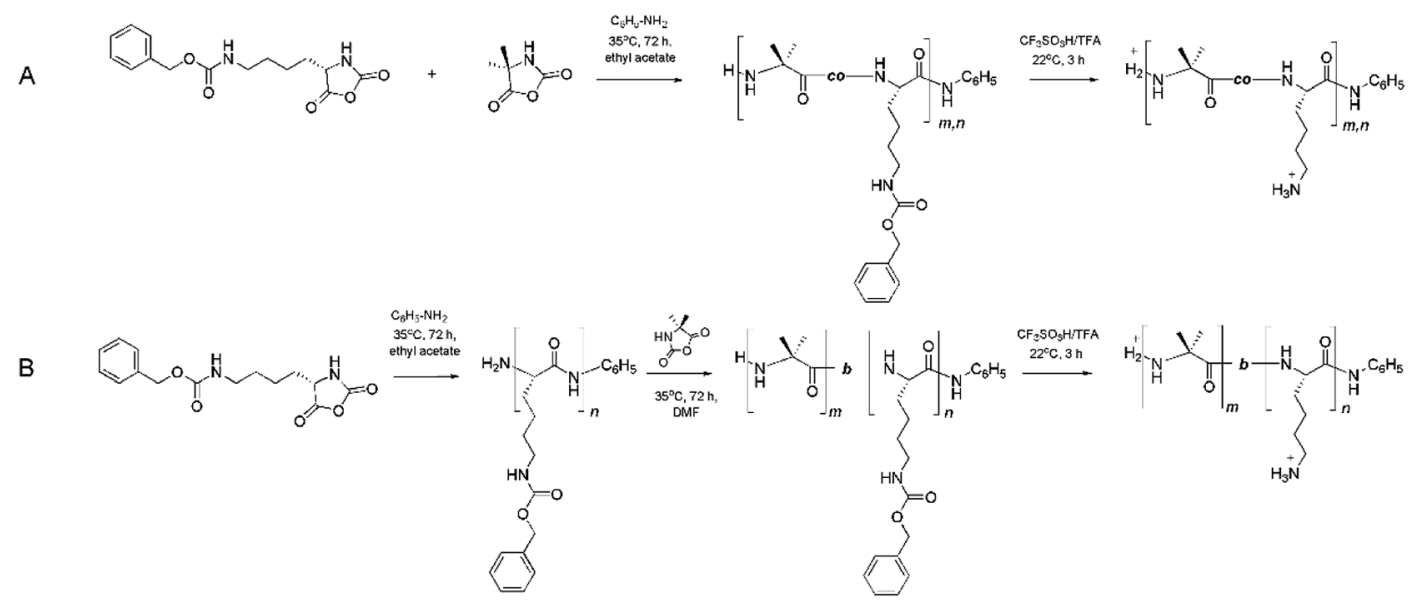

Scheme 1 Strategy for the synthesis of random (A) and block (B) copolymers based on the Lys and Aib units. 
Table 1 Characteristics of the Aib/Lys random copolymers prepared via NCA ROP at different monomer ratios

\begin{tabular}{|c|c|c|c|c|c|c|c|c|}
\hline & & $\begin{array}{l}\text { Calc } \\
\text { com } \\
\text { mold }\end{array}$ & & \multirow[b]{2}{*}{$M_{\mathrm{n}}{ }^{a} \mathrm{P}[\operatorname{Aib}-c o-\operatorname{Lys}(\mathrm{Z})]$} & \multirow[b]{2}{*}{$D$} & \multirow[b]{2}{*}{$\begin{array}{l}{[\text { Lys }] /[\text { Aib }] \text { ratio }} \\
\text { in polypeptides }{ }^{b}\end{array}$} & \multicolumn{2}{|c|}{$\begin{array}{l}\text { Determined } \\
\text { composition }^{b} \\
\text { mol\% }\end{array}$} \\
\hline Sample & $\begin{array}{l}\text { Initial ratio } \\
{[\text { Lys }] /[\text { Aib }]}\end{array}$ & Lys & Aib & & & & Lys & $\mathrm{Aib}$ \\
\hline $\mathrm{R} 1$ & 8 & 89 & 11 & 138000 & 1.67 & 4.5 & 82 & 18 \\
\hline $\mathrm{R} 2$ & 6 & 86 & 14 & 128000 & 1.66 & 4.0 & 80 & 20 \\
\hline $\mathrm{R} 5$ & 1 & 50 & 50 & 54000 & 1.33 & 0.7 & 42 & 58 \\
\hline
\end{tabular}

${ }^{a}$ GPC analysis. ${ }^{b}$ Quantitative RP HPLC amino acid analysis.

Table 2 Characteristics of the PAib ${ }_{m}-b-[\mathrm{PLys}(\mathrm{Z})]_{n}$ block polymers obtained via stepwise NCA ROP

\begin{tabular}{|c|c|c|c|c|c|}
\hline \multirow[b]{2}{*}{ Sample } & \multirow{2}{*}{$\begin{array}{l}{[\text { Aib NCA }] /} \\
{[\text { PLys(Z)] }}\end{array}$} & \multirow{2}{*}{$\begin{array}{l}{[\text { Lys }] /[\text { Aib }] \text { ratio }} \\
\text { in polypeptides }^{a}\end{array}$} & \multicolumn{2}{|c|}{$\begin{array}{l}\text { Determined } \\
\text { composition, mol\% }\end{array}$} & \multirow[b]{2}{*}{$M_{\mathrm{n}}{ }^{b}$ PLys- $b$-PAib } \\
\hline & & & Lys & Aib & \\
\hline B1 & 50 & 1.85 & 70 & 30 & 4200 \\
\hline B2 & 75 & 1.50 & 60 & 40 & 4500 \\
\hline B3 & 100 & 0.96 & 49 & 51 & 5300 \\
\hline
\end{tabular}

molecular weight $\left(M_{\mathrm{n}}\right)=6200(\nexists=1.24)$. The mean number of monomer units in the polypeptide chain was found to be 23 . To vary the length of the hydrophobic $\mathrm{Aib}_{m}$ block, the syntheses of the $\operatorname{PAib}_{m}-b$-[PLys $\left.(\mathrm{Z})\right]_{n}$ polymers were carried out at the $[\mathrm{Aib}$ NCA $] /[$ PLys(Z)] ratios of 50, 75 and 100 (Table 2).

On the contrary to the random Lys(Z)/Aib polymer, the block polypeptides obtained were poorly soluble in many organic solvents and had the tendency to self-aggregate and to form nanoparticles even in DMF, a widely used solvent for polypeptides. Thus, a GPC analysis could not be performed in this case. The polypeptide composition was then calculated from the quantitative RP HPLC amino acid analysis of the hydrolyzed polypeptide samples.

\section{Conformational study}

The information about the peculiarities of the polypeptide 3Dstructures in the solid state was gained by using FT-IR absorption spectroscopy. The frequencies of the IR absorption bands in the amide $\mathrm{I}\left(\mathrm{C}=\mathrm{O}\right.$ stretching, $\left.1600-1700 \mathrm{~cm}^{-1}\right)$, amide II $(\mathrm{N}-$ $\mathrm{H}$ bending and $\mathrm{C}-\mathrm{N}$ stretching, 1480-1575 $\mathrm{cm}^{-1}$ ) and amide $\mathrm{A}$ (N-H stretching, 3300-3500 $\mathrm{cm}^{-1}$ ) regions strongly depend on the overall conformation and the resulting spatial orientation of the peptide units in the backbone. ${ }^{45,46}$ Within each series, either random or block copolymers, the FT-IR absorption spectra show almost identical band patterns, with broad bands due to the presence of a number of co-existing different conformations, whereas a noticeable difference was observed between the two series (Fig. 1; the data are shown for the samples R2, R4, B2 and B3).
Very broad amide A bands in the FT-IR absorption spectra of the lyophilized R2, R4, B2 and B3 copolymers are indicative of wide inter- and intramolecular H-bond networks. The detailed analysis of the amide I and II bands in the spectra of the random copolymers allowed identifying several individual components that could be assigned to various conformations. In particular, the bands centered at about 1650 and $1540 \mathrm{~cm}^{-1}$ suggest the co-existence of random coil and helical conformations, the latter due to the presence of a large amount of the strong helix promoter Aib (Fig. 1, data are shown for the sample R4). ${ }^{47,48}$ Nevertheless, due to the broadness of the FT-IR absorption bands, a noticeable amount of the $\beta$-sheet conformation can be also present in the copolymer structure. On the contrary, the analysis of the amide I structure of the block polypeptides showed the features typical for the $\beta$-sheet conformation: a strong main band at $1625 \mathrm{~cm}^{-1}$ and weaker bands at 1665 and $1690 \mathrm{~cm}^{-1}$, resembling those of spray-dried (Lys $)_{n}$ in the solid state. ${ }^{49}$ Apparently, the presence of Aib in the random co-polymers prevents $\beta$-sheet formation and disrupts the aggregation. This fact can also explain the higher solubility of the random copolymers with respect to the block copolymers.

The CD analysis showed noticeable differences in the copolymer secondary structures, which depended on the Lys/ Aib ratio (Fig. 2).

The lower solubility of the block copolymers, with formation of opalescent solutions, resulted also in lower intensities of the CD spectra as compared to those of the random co-polymers. In water, at neutral or low $\mathrm{pH}$, all copolymers were in random conformation because of the repulsion of the positively charged amino groups. 

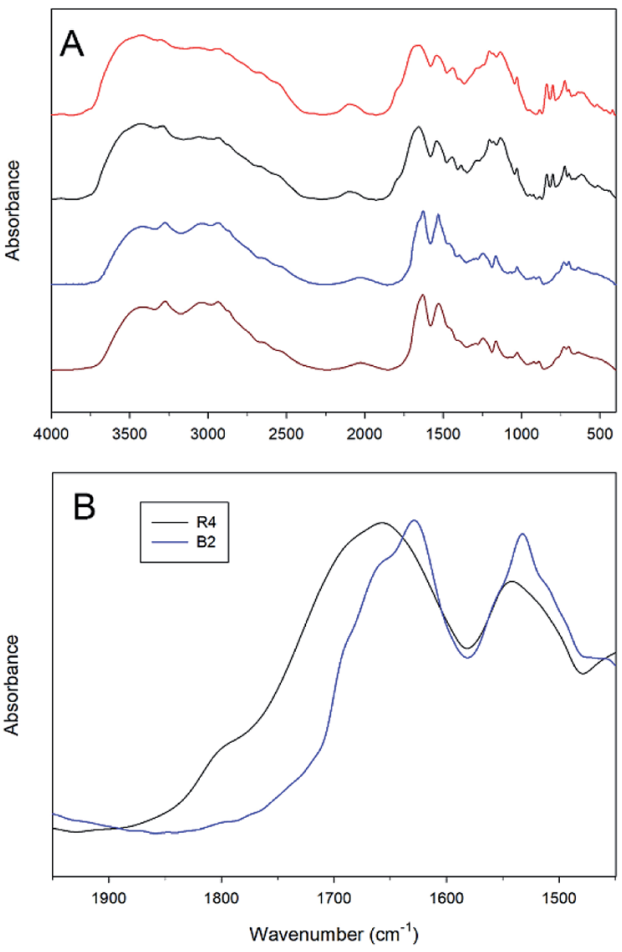

Fig. 1 FT-IR absorption spectra of lyophilized powders of the R2, R4, B2 and B3 copolymers ((A) from top to bottom) and amide I and II bands of R4 and B2 (B).

On the contrary, in a water/TFE mixtures (the latter solvent is known to induce helix formation in peptides ${ }^{50}$ all spectra showed the CD pattern typical for helical conformations, even in the case of the copolymers B2 and B3, forming $\beta$-sheets in lyophilized powders. However, in the spectra of B2 and B3 a noticeable contribution of $\beta$-sheet structure was evidenced by the more intense band at about $222 \mathrm{~nm}$ as compared to that at about $207 \mathrm{~nm}$. The ratio $\Theta_{222} / \Theta_{207}$ decreased and the helix content increased with increasing amount of helix-inducing Aib residues in the copolymers (samples R4 and B3). ${ }^{51}$ Thus, it can be expected, that the composition of the polypeptides and their tendency to form $\beta$-sheets could influence their propensity to form aggregates and further to self-assemble into supramolecular structures.

\section{Preparation and characterization of the nanoparticles}

After removal of $\mathrm{Z}$ protecting group the polypeptides became amphiphilic and self-assembled into supramolecular structures in aqueous media with addition of the organic solvent. Both random and block polypeptides were used for the preparation of nanoparticles. To this aim, the gradual phase-inversion approach (from organic to aqueous medium) was used. In our previous studies on PLys- $b$-PLeu ${ }^{52}$ and PLys- $b$-PIle block copolymers we found that for Lys-based polypeptides the acidic conditions were more suitable for self-assembly..$^{53}$ Apparently, formation of particles with smaller size at $\mathrm{pH} 3$, associated with a more extensive protonation of the Lys side chains, imparts higher solubility to the polypeptides. The subsequent transfer of the nanospheres from the acidic pH to a PBS environment at pH 7.4 did not influence the particle size. ${ }^{53}$ Thus, a $0.1 \mathrm{M} \mathrm{Na-}$ citric buffer, $\mathrm{pH}$ 3.0, was chosen for the preparation of Lys/Aib block and random polypeptide nanoparticles.

Obviously, the differences in the distribution of hydrophilic and hydrophobic units along the macromolecule chain may affect the morphology of the self-assembled particles. Usually, a random distribution of hydrophilic and hydrophobic residues favors the formation of micelles. ${ }^{54}$ In turn, macromolecules with block structures form polymersomes (liposome-like particles or vesicles), as well as micelles. ${ }^{6}$ Indeed, our detailed TEM study of the particles prepared from random and block polypeptides allowed us to conclude that nanospheres of different morphologies do form (Fig. 3).

The nanospheres from random polypeptides were contrasted uniformly with uranyl acetate. On the contrary, the nanospheres from block polypeptides had a dark envelope and a bright core. Thus, in the first case, formation of micelles took place, whereas in the second case the self-assembly generated vesicles. The comparison of the $\mathrm{CD}$ spectra in the organic solvent/water mixtures with the morphology of the forming nanoparticles
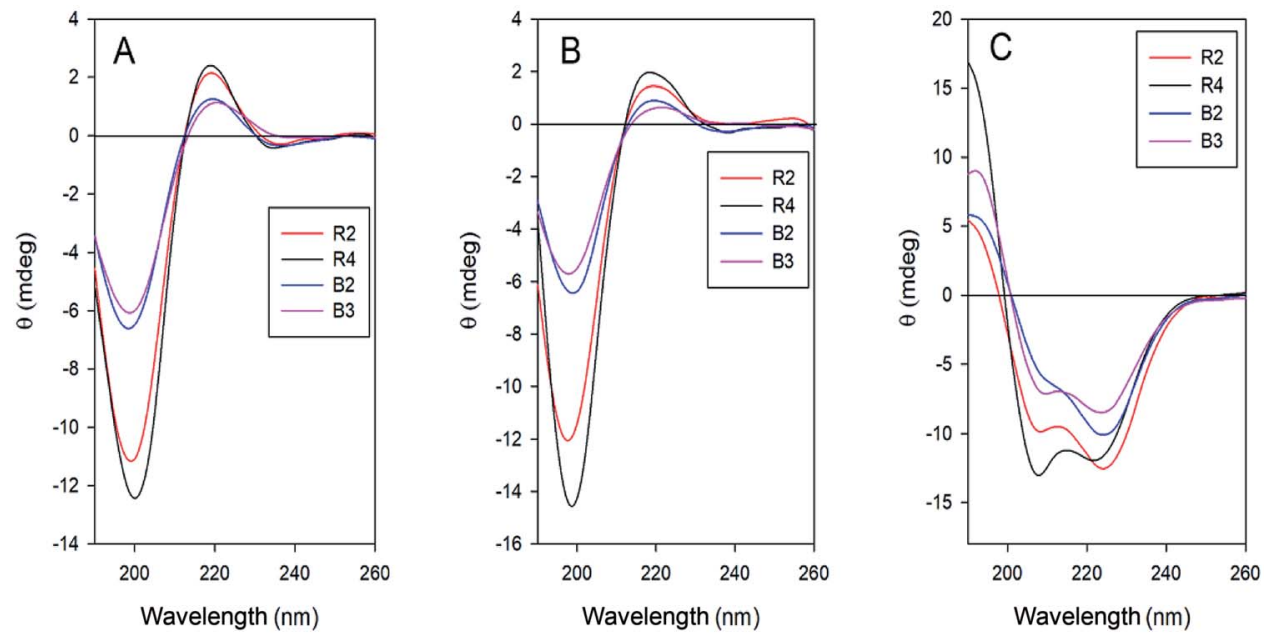

Fig. 2 CD spectra of the copolymers R2, R4, B2 and B3 in water (A), HCl solution, pH 3, (B) and TFE/water mixture (3/1 v/v) (C). 


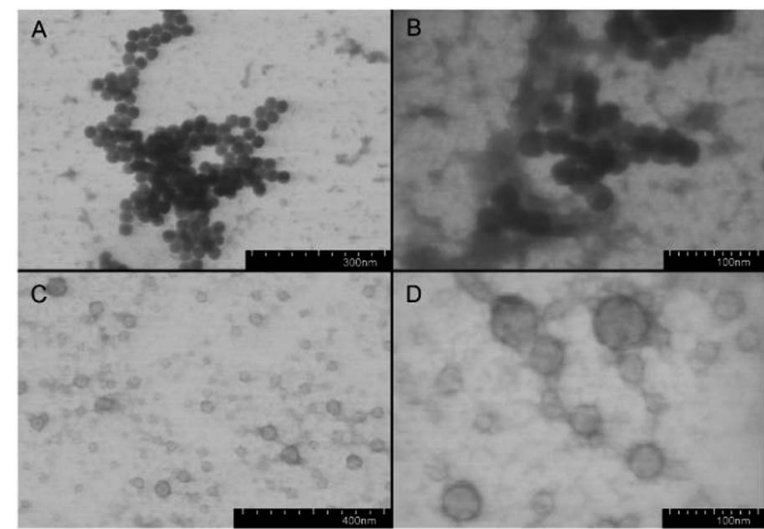

Fig. 3 TEM images of nanoparticles formed by $\mathrm{P}$ (Aib-co-Lys) random copolymer ( $(A$ and B) sample R4) and PAib- $b$-PLys block copolymer ((C and D) sample B2).

Table 3 Results of the DLS measurements of P(Aib-co-Lys) and PAibb-PLys nanoparticles formed at $\mathrm{pH} 3.0$

\begin{tabular}{lll}
\hline Sample & $\begin{array}{l}\text { Hydrodynamic } \\
\text { diameter, } \mathrm{nm}\end{array}$ & PDI \\
\hline P(Aib-co-Lys) & & \\
R1 & $294 \pm 127$ & 0.20 \\
R2 & $312 \pm 138$ & 0.20 \\
R3 & $310 \pm 151$ & 0.24 \\
R4 & $238 \pm 101$ & 0.18 \\
R5 & $185 \pm 87$ & 0.22 \\
PAib-b-PLys & & \\
B1 & & 0.15 \\
B2 & $216 \pm 84$ & 0.23 \\
B3 & $238 \pm 137$ & 0.24 \\
\hline
\end{tabular}

allows to conclude, that not only $\beta$-structure content, but also the distribution of $\beta$-sheets along the polypeptide chain can influence the self-assembly.

Table 3 summarizes the results of the measurements of the hydrodynamic diameter $\left(d_{\mathrm{H}}\right)$ and the PDI of the nanoparticles. The nanoparticles obtained had unimodal size distribution with a mean hydrodynamic diameter from approx. 200 to $400 \mathrm{~nm}$ (depending on the polymer type and composition) and with PDI ranging from 0.15 to 0.24 . In the case of random polypeptides, $d_{H}$ for R1-R3, that contained $18-24 \mathrm{~mol} \%$ of Aib monomer, was about $300 \mathrm{~nm}$. Interestingly, the increase of Aib amount up to $58 \mathrm{~mol} \%$ for R5 induced a decrease in $d_{\mathrm{H}}$. This finding can be explained by the tight folding of the hydrophobic part (Aib stretch) of the macromolecule in aqueous environments. Another reason for this phenomenon can be the diminished repulsion among the less abundant, positively charged monomers. The discrepancy of the data acquired by TEM and DLS methods originates from the different principles of the measurement. DLS detects hydrodynamic diameter of the particles with polymeric chains exposed to the solvent, and it is obviously higher than that determined for the dried samples in TEM. ${ }^{55,56}$
While the amphiphilic random copolymers self-assembled into micelles, with a compact hydrophobic core, the amphiphilic block-copolymers formed vesicle-like structures containing a hydrophobic membrane and an aqueous interior. For the block polypeptides the increase of the hydrophobic part was accompanied by an increase of their hydrodynamic diameter. A similar result was previously observed for PGlu- $b$-PPhe polymersomes. ${ }^{57}$ The most probable reason for such effect is the decrease of polypeptide solubility because of its increased hydrophobicity. Therefore, the higher self-aggregation caused by the hydrophobic interactions leads to a reduction of the particle core.

\section{Biodegradation study}

In vitro biodegradation studies represent a complicated task as usually the degradation rate differs from that of the in vivo process because of the complex composition of the biological fluids containing a number of highly active enzymes. Nevertheless, the results of the in vitro degradation allow a comparative analysis of the biodegradability of different biomaterials. ${ }^{58}$

To evaluate the biodegradation of the prepared nanoparticles, the representative samples $\mathrm{R} 1, \mathrm{R} 4$ and $\mathrm{B} 2$ were selected. R4 and B2 were chosen due to the similarity of the amino acid ratio and size after self-assembly. R1 sample was added for the comparison with R4 to evaluate the effect of hydrophobic to hydrophilic amino acid ratio on biodegradation of random copolymers. To assess the contribution of Aib to the biodegradation rate, PLys- $b$-PLeu nanoparticles were used as well. Papain was selected as a biocatalyst. This proteolytic enzyme works at physiological conditions ( $\mathrm{pH} 7.4$ and $37^{\circ} \mathrm{C}$ ) and cleaves the peptide bonds between numerous $\alpha$-amino acids.

During the 35 day biodegradation experiments, the hydrodynamic diameters of the particles were monitored by DLS (Fig. 4). Except R1, containing the highest ratio of hydrophilic to hydrophobic amino acids, the degradation of the polypeptides (R4, B2 and PLys-b-PLeu) was accompanied by the increase in the particle size after the first day of degradation process and a slow decrease of the size within further two weeks. Such surge

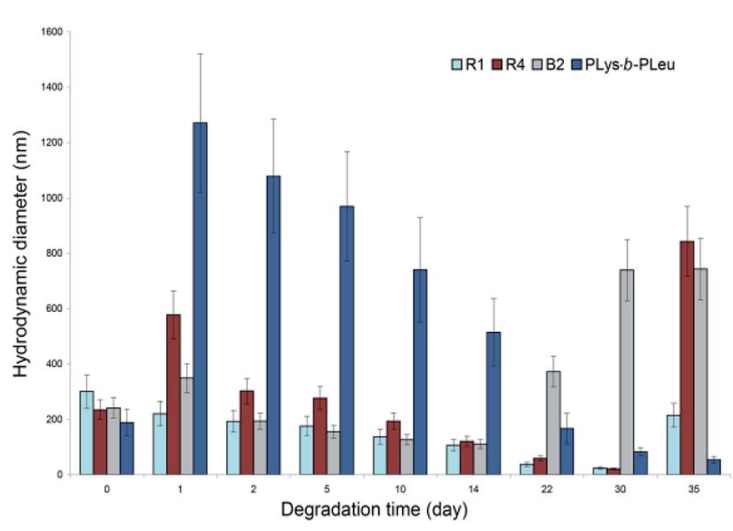

Fig. 4 Changes in the hydrodynamic sizes of polypeptide particles during the biodegradation processes induced by papain (0.01 M PBS, $\left.\mathrm{pH} 7.4,37^{\circ} \mathrm{C}\right)$. 
in the particle size is caused by the partial degradation of the hydrophilic Lys-based shell and the exposure of the hydrophobic part to the solvent. In turn, this latter event favored the aggregation of the particles because of the hydrophobic interactions in the aqueous medium. The highest aggregation and, consequently, the highest burst in size was observed for the polypeptide based on coded amino acids, e.g. Lys and Leu. This peptide was used as benchmark for the degradation studies. In this case, the fast degradation of the (Lys) ${ }_{n}$ block led to the quick aggregation of the nanoparticles after a day of coincubation with the enzyme. A considerable aggregation of the particles formed by Aib-containing polypeptides was observed after co-incubation with the enzyme within three weeks for PAib-b-PLys and within a month for random polypeptides.

The HPLC amino acid analysis allowed identification of free amino acids released in the reaction media during the degradation process. On the contrary to PLys- $b$-PLeu degradation, where Leu was detected already after one day of biodegradation, free Aib was found after 5 days for R4 and B2 and 9 days for R1. Moreover, the analysis of free amino acid content after 14 and 35 days revealed that Lys accumulation for both PLys- $b$-PLeu and B2 was faster than that of the random polypeptides R1 and R4 (Fig. 5A). In case of Aib, the slowest accumulation of the amino acid was observed for B2 sample. It indicates the slowest degradation rate of hydrophobic block of B2 copolymer in comparison with PLys- $b$-PLeu, as well as with R1 and R4 samples (Fig. 5B). This behavior can be explained by the fast degradation of Lys stretches by papain, whereas Aib sequence can be hardly targeted by the hydrolytic enzyme.

The accumulation of free amino acids produced during the degradation of random Aib-containing polypeptides was
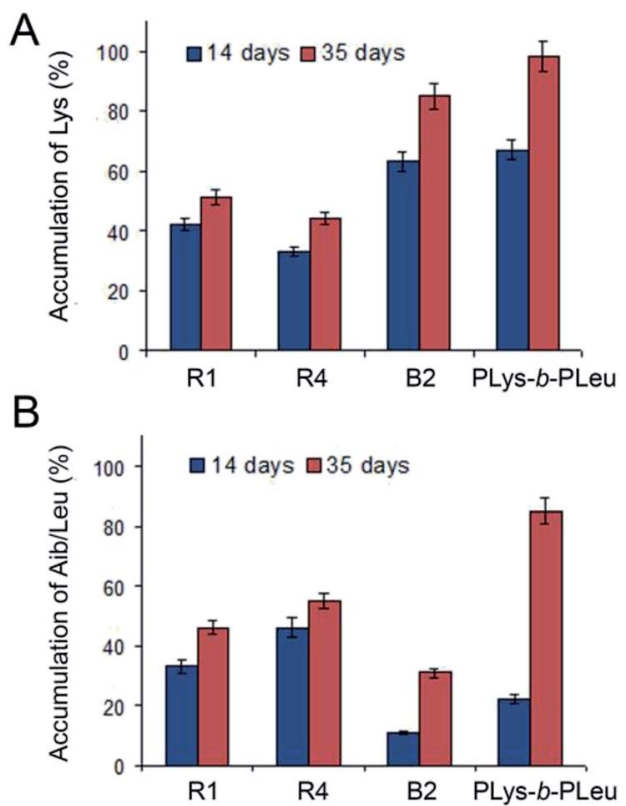

Fig. 5 Accumulation of free Lys (A) and Aib/Leu (B) during the biodegradation process of random and block copolymers based on Lys and Aib, as well as PLys- $b$-PLeu induced by papain (0.01 M PBS, pH $\left.7.4,37^{\circ} \mathrm{C}\right)$. comparable for R1- and R4-based particles, but it was slower than that observed for the benchmark polypeptide as well.

\section{Cell experiments}

The nanoparticles obtained were tested in cell experiments using the HepG2 cell line.

The results on the cell viability determined by the MTT assay are presented in Fig. 6. Up to the concentration $0.125 \mathrm{mg} \mathrm{mL}^{-1}$, cell viability for nanoparticles based on both kinds of copolymers was higher than $80 \%$. However, at concentration of $0.25 \mathrm{mg} \mathrm{mL}^{-1}$ the cytotoxicity of the random copolymers was twice higher than that of the block copolymers. The EC50 values for the tested random (R4) and block-copolymer (B2) were 0.170 and 0.245 , respectively.

The lower cytotoxicity of the nanomaterials based on block copolymers can be related to the better burial of the hydrophobic residues inside the particles. This behavior, in turn, favors the stabilization of the particles in the culture medium. In addition, the exposed hydrophobic residues of the random polymers can favor the membrane disrupting activity of the nanostructures.

To study the cellular uptake of Lys/Aib-based nanomaterials, the fluorescent microscopy technique was applied. Samples R4 and B2 were covalently labeled by use of the Cy3 dye and incubated with HepG 2 cells for 4 hours. When the incubation time was over the cell nuclei were stained with DAPI. The internalization of both kinds of nanoparticles indicated a relatively uniform distribution of Cy3 (red) in the cytoplasm around the nucleus (blue) (Fig. 7).

The kinetics of internalization was studied via incubation of Cy3-labeled nanoparticles with HepG2 for 48 hours. The fluorescent signal for both kinds of nanoparticles (series $\mathrm{R}$ and $\mathrm{B}$ ) considerably increased during the initial hours and reached the maximum after 24 hours of incubation (Fig. 8). The difference in fluorescence intensity can be explained by different amount of Cy3 label covalently bound to the nanoparticle surface. In case of nanoparticles formed by random polymers (R4 sample) the labeling efficiency is much higher because most hydrophilic lysine residues are exposed to aqueous surrounding and accessible for the reaction with the dye. In turn, for polymersomes (B2 sample), which have bilayer polymer membrane,

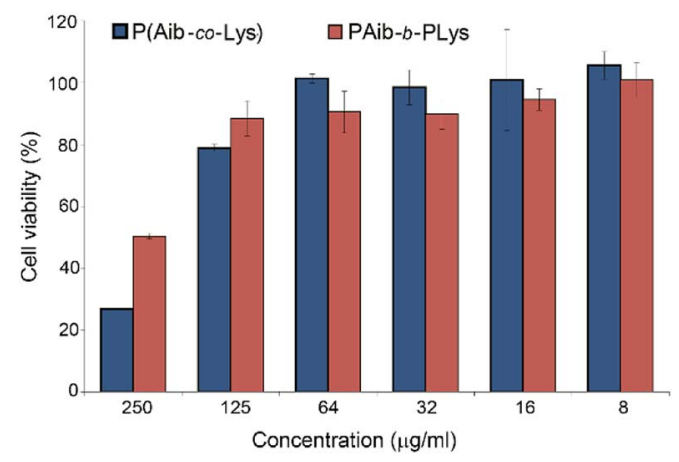

Fig. 6 Cell viability (HepG2) in the presence of P(Aib-co-Lys) (sample R4) and PAib-b-PLys (sample B2) during 48 hours. 

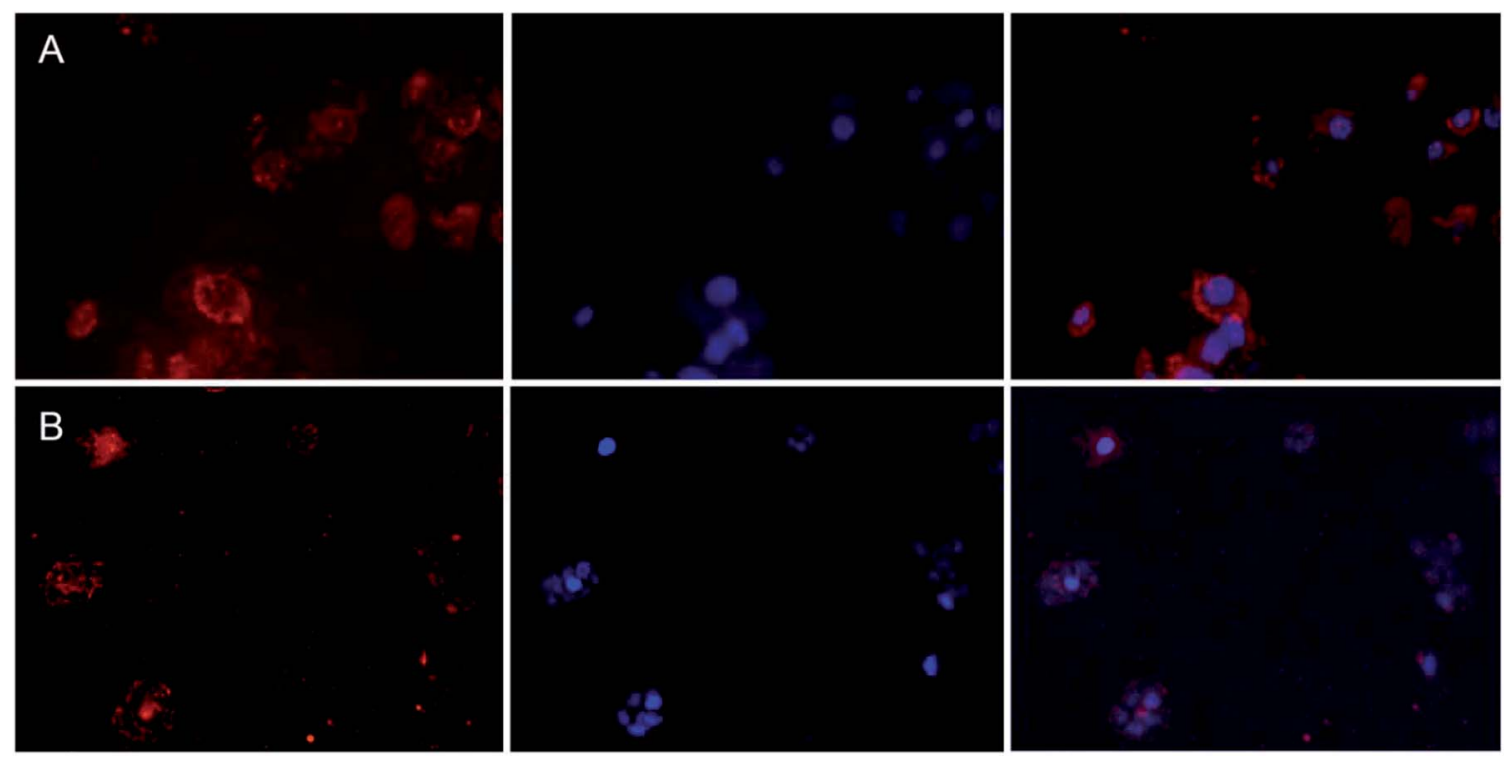

Fig. 7 Fluorescent images $(\times 20)$ of HepG2 cells treated with Cy3-labeled polypeptides $\left(60 \mu \mathrm{g} \mathrm{mL}{ }^{-1}\right)$ : (A) P(Lys-co-Aib) (sample R4); (B) PAib- $b$ PLys (sample B2). Each image comprises (from left to right) Cy3, DAPI and merged fluorescence.

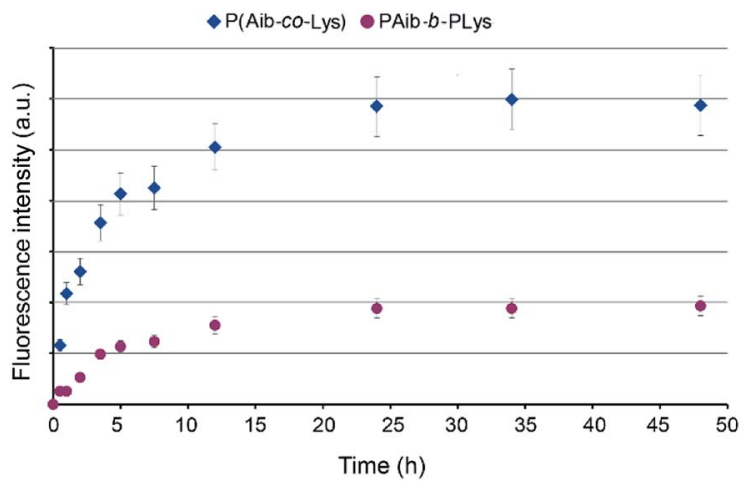

Fig. 8 Kinetics of cell uptake of Cy3-labeled P(Aib-co-Lys) (sample R4) and PAib-b-PLys (sample B2) nanoparticles (concentration: $60 \mu \mathrm{g}$ $\mathrm{mL}^{-1}$ ).

about a half of lysines are buried inside the particle. Thus, for polymersomes the lower amount of lysine units is accessible for modification.

\section{Conclusions}

A series of random and block copolypeptides based on Lys and Aib was synthesized via ROP NCA. The random polypeptides, lyophilized from water, adopted both random coil and $\alpha$-helical conformations, whereas the block-type polypeptides showed a high content of $\beta$-sheets in their structure. In water, at neutral or low $\mathrm{pH}$, all copolymers were unordered because of the repulsion of the positively charged side chains of Lys residues. On the contrary, in TFE all spectra revealed a pattern typical for helical conformations, even in the case of block copolymers with high $\beta$-sheet propensity. As expected, the helix content increased with increasing amount of the helix-inducing Aib residue in the polypeptides. All the copolymers formed nanoparticles of different morphology, which depended on the distribution of the Aib residues (random or block) along the peptide chain. In particular, random polypeptides selfassembled into micelles, whereas the block-type polypeptides formed vesicles. At optimal conditions, the nanoparticles obtained showed an almost unimodal size distribution with mean hydrodynamic diameter of 200-400 nm. By increasing the Aib amount, the hydrodynamic diameter of the particles based on random copolymer decreased, probably, because of the increasing self-aggregation and the reduction of the particle core. These results can be important for the further understanding of the peculiarities of the long Aib polypeptides folding and the influence of the secondary structure on of the morphology of the resulting nanoparticles. Aib-containing nanoparticles proved to be more stable to enzymatic degradation than those based on only coded amino acids. They did not induce cell death up to the concentration $0.125 \mathrm{mg} \mathrm{mL}^{-1}$ and were internalized into the cell cytoplasm within 24 hours. Due to all these features the novel, bio-inspired polypeptide nanomaterials described herein can find various biomedical applications, such as development of improved drug delivery formulations, fillers for the scaffolds, etc.

\section{Conflicts of interest}

There are no conflicts to declare.

\section{Acknowledgements}

The chemical synthesis and biological evaluation of amphiphilic block copolymers was supported by a grant of the Russian Science Foundation (\#14.50.00069). The authors also thank the Chemical Analysis and Materials Research Centre of St Petersburg State University for the HPLC amino acid analysis. B. B. 
and F. F. are grateful to the Italian Ministry of Research for financial support (PRIN 2015, Prot. 20157WW5EH).

\section{References}

1 C. Ding and Z. Li, Mater. Sci. Eng., C, 2017, 76, 1440.

2 J. Li, X. Chang, X. Chen, Z. Gu, F. Zhao, Z. Chai and Y. Zhao, Biotechnol. Adv., 2014, 32, 727.

3 J. Wang, C. Gong, Y. Wang and G. Wu, RSC Adv., 2014, 4, 15856.

4 M. S. Shim and Y. J. Kwon, Adv. Drug Delivery Rev., 2012, 64, 1046.

5 I. Guryanov, S. Fiorucci and T. Tennikova, Mater. Sci. Eng., C, 2016, 68, 890.

6 S. Kumar and P. Maiti, RSC Adv., 2016, 6, 67449.

7 K. Letchford and H. Burt, Eur. J. Pharm. Biopharm., 2007, 65, 259.

8 J. S. Lee and J. Feijen, J. Controlled Release, 2012, 161, 473.

9 N. Habibi, N. Kamaly, A. Memic and H. Shafiee, Nano Today, 2016, 11, 41.

10 K. Matsuurua, RSC Adv., 2014, 4, 2942.

11 A. V. Hubina, A. A. Pogodaev, V. V. Sharoyko, E. G. Vlakh and T. B. Tennikova, React. Funct. Polym., 2016, 100, 173.

12 J. Wang, K. Liu, R. Xing and X. Yan, Chem. Soc. Rev., 2016, 45, 5589.

13 Z. Song, Z. Han, S. Lv, C. Chen, L. Chen, L. Yin and J. Cheng, Chem. Soc. Rev., 2017, 46, 6570.

14 V. Mikhalevich, I. Craciun, M. Kyropoulou, C. G. Palivan and W. Meier, Biomacromolecules, 2017, 18, 3471.

15 N. Stoppacher, N. K. N. Neumann, L. Burgstaller, S. Zeilinger, T. Degenkolb, H. Brückner and R. Schuhmacher, Chem. Biodivers., 2013, 10, 734.

16 Peptaibiotics, ed. C. Toniolo and H. Bruckner, Wiley-VCH, Weinheim, Germany, 2009.

17 M. Venanzi, E. Gatto, F. Formaggio and C. Toniolo, J. Pept. Sci., 2017, 23, 104.

18 I. L. Karle and P. Balaram, Biochemistry, 1990, 29, 6747.

19 C. Toniolo, M. Crisma, F. Formaggio and C. Peggion, Biopolymers, 2001, 60, 396.

20 E. Longo, A. Moretto, F. Formaggio and C. Toniolo, Chirality, 2011, 23, 756.

21 S. Zikou, A.-I. Koukkou, P. Mastora, M. Sakarellos-Daitsiotis, C. Sakarellos, C. Drainas and E. Panou-Pomonis, J. Pept. Sci., 2007, 13, 481.

22 K. Kono, H. Nishii and T. Takagishi, Biochim. Biophys. Acta, 1993, 1164, 81.

23 L. Garbuio, S. Antonello, I. Guryanov, Y. Li, M. Ruzzi, N. J. Turro and F. Maran, J. Am. Chem. Soc., 2012, 134, 10628.

24 R. A. G. D. Silva, S. C. Yasui, J. Kubelka, F. Formaggio, M. Crisma, C. Toniolo and T. A. Keiderling, Biopolymers, 2002, 65, 299.

25 P. Hanson, G. Millhauser, F. Formaggio, M. Crisma and C. Toniolo, J. Am. Chem. Soc., 1996, 118, 7618.

26 S. Mondal, M. Varenik, D. N. Bloch, Y. Atsmon-Raz, G. Jacoby, L. Adler-Abramovich, L. J. W. Shimon, R. Beck, Y. Miller, O. Regev and E. Gazit, Nat. Commun., 2017, 8, 14018.
27 S. Grubičić, B. Chandramouli, V. Barone and G. Brancato, Phys. Chem. Chem. Phys., 2016, 18, 20389.

28 M. Konda, S. Bhowmik, S. M. Mobin, S. Biswas and A. K. Das, ChemistrySelect, 2016, 1, 2586.

29 P. Gobbo, S. Antonello, I. Guryanov, F. Polo, A. Soldà, F. Zen and F. Maran, ChemElectroChem, 2016, 3, 2063.

30 S. Mondal, L. Adler-Abramovich, A. Lampel, Y. Bram, S. Lipstman and E. Gazit, Nat. Commun., 2015, 6, 8615.

31 M. Ueda, A. Makino, T. Imai, J. Sugiyama and S. Kimura, J. Pept. Sci., 2011, 17, 94.

32 A. Pramanik, A. Paikar, T. Das, K. Maji and D. Haldar, RSC Adv., 2016, 6, 39172.

33 Synthesis of peptides and peptidomimetics, Houben-Weyl: Methods of Organic Chemistry, ed. M. Goodman, A. Felix, L. Moroder and C. Toniolo, vol. E 22, Thieme, Stuttgart, Germany, 2003.

34 A. Takasu, S. Horikoshi and T. Hirabayashi, Biomacromolecules, 2005, 6, 2334.

35 M. De Zotti, B. Biondi, C. Peggion, F. Formaggio, Y. Park, K.-S. Hahm and C. Toniolo, Org. Biomol. Chem., 2012, 10, 1285.

36 K. B. H. Salah and N. Inguimbert, Org. Lett., 2014, 16, 1783. 37 A. Siow, K. Hung, P. W. R. Harris and M. A. Brimble, Eur. J. Org. Chem., 2017, 350.

38 K. Tsuchiya and K. Numata, Chem. Commun., 2017, 53, 7318. 39 M. Ueda, A. Makino, T. Imai, J. Sugiyama and S. Kimura, Polym. J., 2013, 45, 509.

40 M. Ueda, A. Uesaka and S. Kimura, Chem. Commun., 2015, 51, 1601.

41 A. Paikar, A. Pramanik, T. Das and D. Haldar, Polym. Chem., 2017, 8, 396.

42 J. Cheng and T. J. Deming, Top. Curr. Chem., 2012, 310, 1.

43 E. Fröhlich, Int. J. Nanomed., 2012, 7, 5577.

44 R. Wilder and S. Mobashery, J. Org. Chem., 1992, 57, 2755.

45 M. Jackson and H. H. Mantsch, Crit. Rev. Biochem. Mol. Biol., 1995, 30, 95.

46 A. Mirtič and J. Grdadolnik, Biophys. Chem., 2013, 175-176, 47.

47 I. Guryanov, F. Polo, E. V Ubyvovk, E. Korzhikova-Vlakh, T. Tennikova, A. T. Rad, M.-P. Nieh and F. Maran, Chem. Sci., 2017, 8, 3228.

48 S. J. Prestrelski, N. Tedeschi, T. Arakawa and J. F. Carpenter, Biophys. J., 1993, 65, 661.

49 A. Mauerer and G. Lee, Eur. J. Pharm. Biopharm., 2006, 62, 131.

50 M. Goodman, A. S. Verdini, C. Toniolo, W. D. Phillips and F. A. Bovey, Proc. Natl. Acad. Sci. U. S. A., 1969, 64, 444.

51 C. Toniolo, A. Polese, F. Formaggio, M. Crisma and J. Kamphuis, J. Am. Chem. Soc., 1996, 118, 2744.

52 N. N. Zashikhina, M. V. Volokitina, V. A. Korzhikov-Vlakh, I. I. Tarasenko, A. Lavrentieva, T. Scheper, E. Rühl, R. V. Orlova, T. B. Tennikova and E. G. Korzhikova-Vlakh, Eur. J. Pharm. Sci., 2017, 109, 1.

53 E. G. Vlakh, E. V. Grachova, D. D. Zhukovsky, A. V. Hubina, A. S. Mikhailova, J. R. Shakirova, V. V. Sharoyko, S. P. Tunik and T. B. Tennikova, Sci. Rep., 2017, 7, 41991. 
54 Functional Monomers and Polymers, ed. K. Takemoto, R. M. Ottenbrite and M. Kamachi, Marcel Dekker, NY, 2nd edn, 1997.

55 A. O. Moughton, J. P. Patterson and R. K. O'Reilly, Chem. Commun., 2011, 47, 355.

56 H. R. Marsden, L. Gabrielli and A. Kros, Polym. Chem., 2010, 1,1512 .
57 E. Vlakh, A. Ananyan, N. Zashikhina, A. Hubina, A. Pogodaev, M. Volokitina, V. Sharoyko and T. Tennikova, Polymers, 2016, 8, 212.

58 H. S. Azevedo and R. L. Reis, Understanding the enzymatic degradation of biodegradable polymers and strategies to control their degradation rate, in Biodegrad. Syst. Tissue Eng. Regen. Med., ed. R. L. Reis and J. S. Roman, CRC Press, Boca Raton, 2004, pp. 177-201. 